\title{
Evolution of Computed Tomography Findings in Secondary Aortoenteric Fistula
}

\author{
Ahmet Bas ${ }^{1}$; Osman Simsek ${ }^{2}$; Sedat Giray Kandemirli ${ }^{1,{ }^{*}}$; Babak Rafiee ${ }^{1}$; Fatih Gulsen ${ }^{1}$; \\ Furuzan Numan ${ }^{1}$ \\ ${ }^{1}$ Department of Radiology, Faculty of Medicine, Cerrahpasa University, Istanbul, Turkey \\ ${ }^{2}$ Department of General Surgery, Faculty of Medicine, Cerrahpasa University, Istanbul, Turkey \\ ${ }^{*}$ Corresponding author: Sedat Giray Kandemirli, Department of Radiology, Faculty of Medicine, Cerrahpasa University, Istanbul, Turkey. Tel: +90-5543971851, Fax: +90-2124143167, \\ E-mail: gskandemirli@yahoo.com
}

Received: August 13, 2014; Revised: September 4, 2014; Accepted: September 20, 2014

\begin{abstract}
Aortoenteric fistula is a rare but significant clinical entity associated with high morbidity and mortality if remain untreated. Clinical presentation and imaging findings may be subtle and prompt diagnosis can be difficult. Herein, we present a patient who initially presented with abdominal pain and computed tomography showed an aortic aneurysm compressing duodenum without any air bubbles. One month later, the patient presented with gastrointestinal bleeding and computed tomography revealed air bubbles within aneurysm. With a diagnosis of aortoenteric fistula, endovascular aneurysm repair was carried out. This case uniquely presented the computed tomography findings in progression of an aneurysm to an aortoenteric fistula.
\end{abstract}

Keywords: Fistula; Endovascular Procedures; Aorta

\section{Introduction}

Aortoenteric fistula (AEF) results from direct communication between the aorta and gastrointestinal (GI) tract. It is a rare but significant clinical entity associated with high morbidity and mortality if remains untreated (1). AEFs can be primary or secondary to an aortic graft placement or surgery. Primary AEF has an incidence rate at autopsy of $0.04 \%$ to $0.07 \%$. Secondary AEFs are complications of aortic vascular procedures with a postoperative incidence rate of $0.5 \%$ to $2.3 \%$ and mortality rate of $65 \%$ to $100 \%(2,3)$. Early diagnosis is essential for a timely intervention to decrease the mortality rates. In this case, we present a patient who initially presented with abdominal pain and developed GI bleeding one month later. Computed tomography (CT) findings showed the progression of an aortic aneurysm adherent to duodenum to an AEF. This case is unique since it presents evolution of CT findings in AEF, which is highly fatal without intervention.

\section{Case Presentation}

A 68-year-old man with a medical history of hypertension, aortobifemoral bypass, and femoro-femoral bypass grafting presented to the emergency department with severe diffuse abdominal pain. The patient was complaining of a vague abdominal pain for the last month that had intensified in the last couple of days. On initial examination, he was hemodynamically stable with a soft non-distended abdomen that was tender to palpation in the left lower quadrant. A slightly elevated white blood cell count of $17.79 \times 10^{9} / \mathrm{L}$ and elevated C- reactive protein level of 236 $\mathrm{mg} / \mathrm{L}$ (normal range, 0-5 mg/L) or $22247 \mathrm{nmol} / \mathrm{L}$ (range, 0.76-28.5 nmol/L) were measured in venous samples. Contrast-enhanced CT revealed extensive atherosclerosis of abdominal aorta and an infrarenal aneurysm 40-mm transverse diameter and 6-cm length (Figure 1). Aneurysm had a slightly hyperdense thrombosed crescentic portion with $11-\mathrm{mm}$ diameter. In continuation with the thrombosed aneurysm, a soft tissue density was extending anteriorly. The soft tissue density was adherent to the duodenum and was slightly compressing it. With a suspected diagnosis of intramural hematoma and contained aneurysm rupture, conventional angiography of aorta was performed. Angiography confirmed abdominal aorta aneurysm and showed no contrast extravasation. The patient was prescribed pentoxifylline and coraspin and was discharged. One month later, the patient presented with mild-to-moderate hematemesis and severe abdominal pain, accompanied by tenderness to palpation with positive rebound in left lower quadrant. A nasogastric tube initially drained only gastric contents. During follow-up, the patient had an episode of melena and positive results in nasogastric lavage. A contrast enhanced CT showed an infrarenal aneurysm $50 \mathrm{~mm}$ in transverse diameter and 6 $\mathrm{cm}$ in length with the progression of mural thrombus to a diameter of $2 \mathrm{~cm}$. Marked compression of small intestine,

Copyright (C) 2015, Tehran University of Medical Sciences and Iranian Society of Radiology. This is an open-access article distributed under the terms of the Creative Commons Attribution-NonCommercial 4.0 International License (http://creativecommons.org/licenses/by-nc/4.0/) which permits copy and redistribute the material just in noncommercial usages, provided the original work is properly cited. 
Bas A et al.
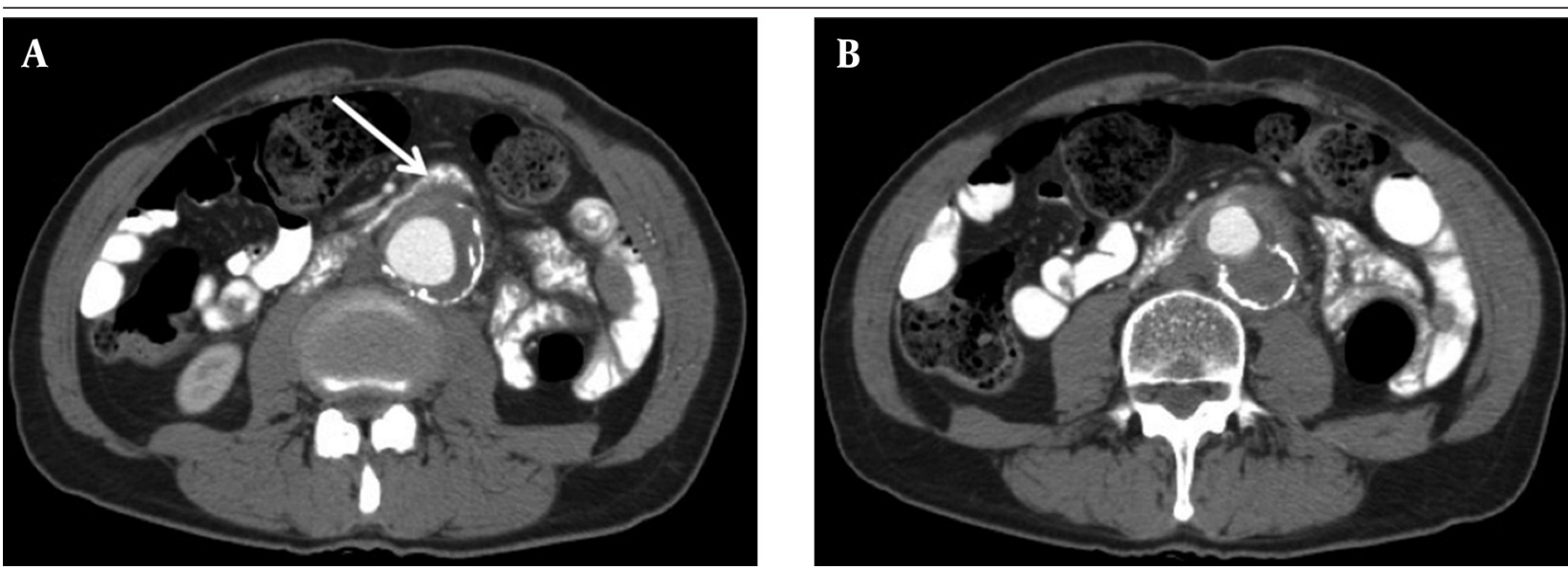

Figure 1. A 68-year-old man with abdominal pain and gastrointestinal bleeding diagnosed as aortoenteric fistula. A,B, Initial contrast-enhanced computed tomography reveals extensive atherosclerosis of abdominal aorta and an infrarenal thrombosed aneurysm. In the extension of the thrombosed aneurysm, a soft tissue density is extending anteriorly (arrow), adherent to the duodenum and slightly compressing it. No air bubbles can be seen.
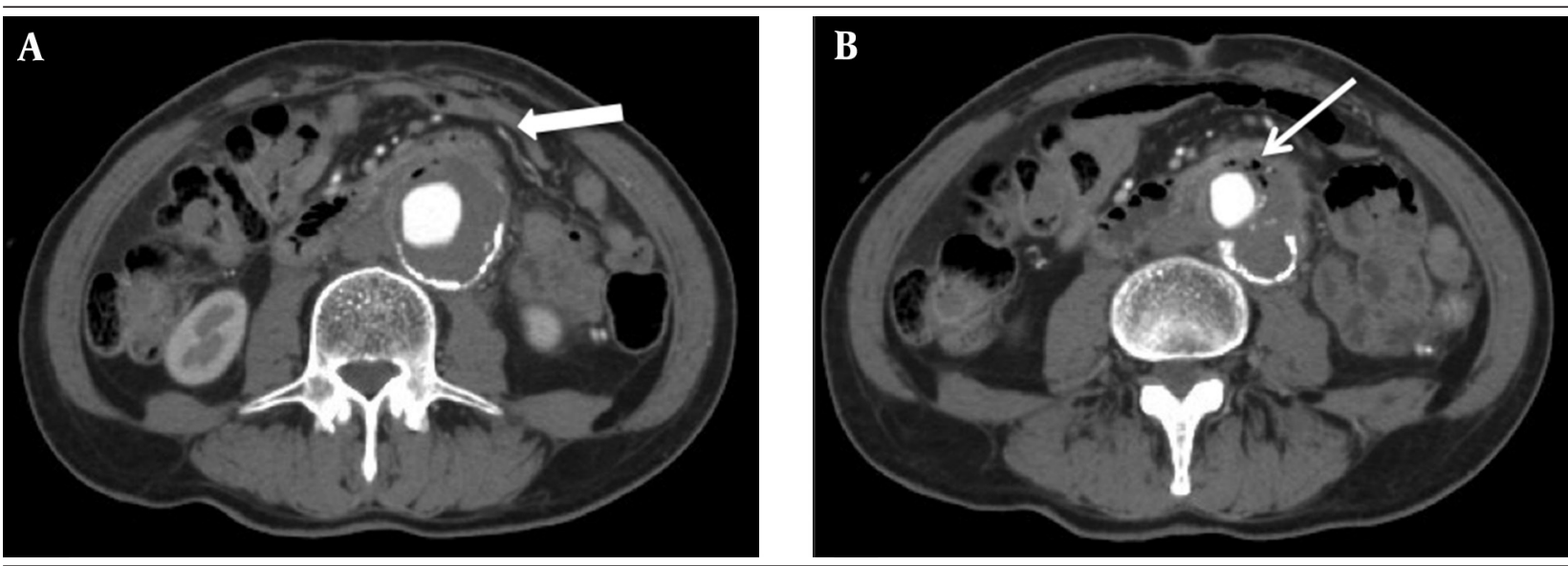

Figure 2. A,B, After melena, contrast-enhanced computed tomography shows slightly increased aneurysm size compared to previous imaging. Marked compression of small intestine, anterosuperior to aneurysm (thick arrow), with small bubbles of air within thrombus (arrow) was noted.
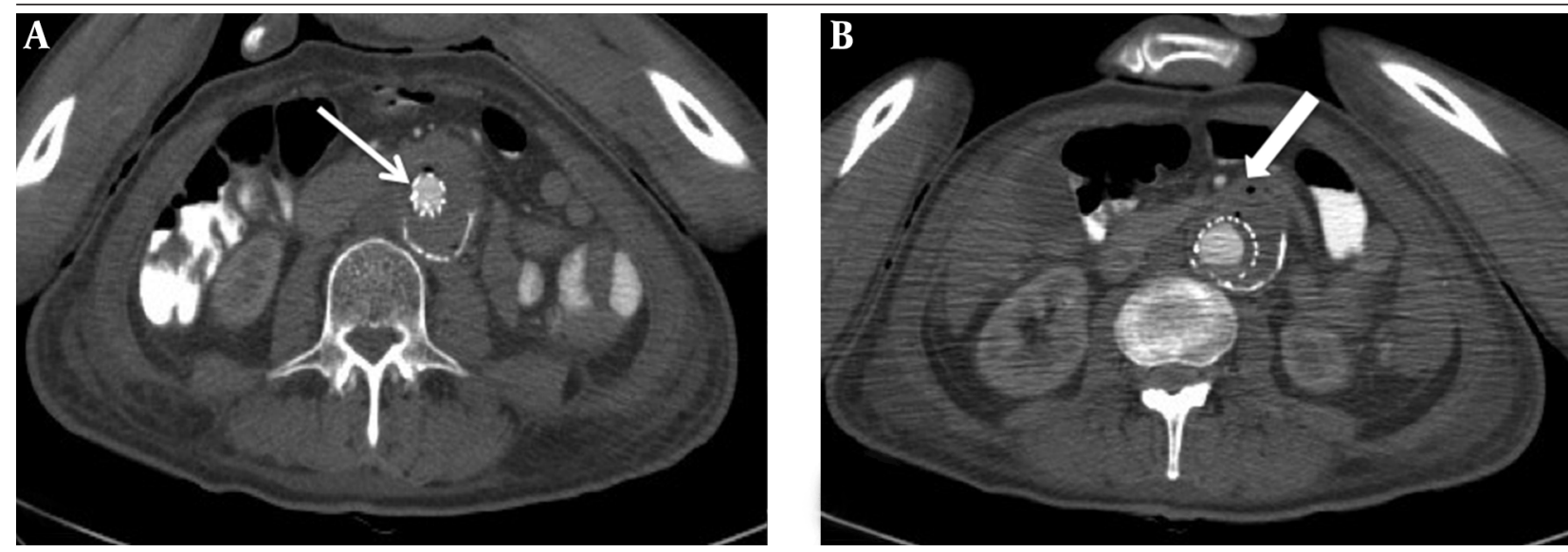

Figure 3. A, B, After endovascular aneurysm repair, contrast-enhanced computed tomography reveals struts of graft (arrow) and regression of aneurysm with a slightly decreased compression on duodenum. Air bubbles (thick arrow) can be seen postoperatively.

anterosuperior to aneurysm, and small air bubbles within thrombus were noted (Figure 2). With a suspected diagnosis of AEF, endovascular aneurysm repair (EVAR) was planned. Two endovascular stent grafts, extending from re- nal arteries origins to right iliac artery, were deployed, followed by balloon dilatation of proximal graft site. Control angiography showed absence of contrast extravasation and endoleak. Prophylactic broad-spectrum antibiotic was 
Bas A et al.

administered. A control contrast-enhanced CT showed regression of aneurysm with a slightly decreased compression on duodenum (Figure 3). Postoperatively, regression of mural thrombus and air bubbles within thrombus were noted. The patient was discharged on antibiotic therapy and died of myocardial infarction nine months later.

\section{Discussion}

\subsection{Pathogenesis}

The AEF is a rare but significant clinical entity associated with high morbidity and mortality if remains untreated. It results from direct communication between the aorta and GI tract. Any segment of GI tract may be involved, but third portion of the duodenum is most commonly involved because of its relatively fixed position and close proximity to the abdominal aorta (1). AEFs can be primary or secondary to an aortic graft placement or surgery. Primary AEF has an incidence rate of $0.04 \%$ to $0.07 \%$ at autopsies. Pathogenesis of primary AEF involves atherosclerotic aneurysm with an elevated amount of metalloproteinases leading to inflammatory cell infiltration and resultant fibrosis. Fibrosis may cause adherence and erosion of aneurysm into enteric wall (4). Secondary AEFs were first described by Brock in 1953 and are complications of aortic vascular procedures with a postoperative incidence rate of $0.5 \%$ to $2.3 \%$ and a median interval of 16 months (range, 4-72 months) $(2,3)$. Fistula forms from prolonged contact of graft to a fixed bowel segment with pressure necrosis and graft eroding the bowel wall. Secondary AEFs are subdivided into type 1 , where direct communication between bowel and aortic lumen is present, and type 2 , where communication between bowel and perigraft region with a luminal connection is present (1).

\subsection{Clinical Presentation and Diagnosis}

Diagnosis of AEF requires a high index of suspicion. Classic triad of primary AEF is seen in $11 \%$ of cases and consists of GI bleeding, abdominal pain, and pulsatile abdominal mass (5). Another classic feature of AEF is herald bleeding, occurring in $30 \%$ of cases. It is a minor bleed preceding the massive exsanguinating bleeding by an interval ranging from six hours to several weeks. It is usually self-limiting due to vasospasm and thrombus formation. Herald bleeding may provide an important diagnostic clue and interval for diagnosis and intervention. Therefore, if a patient with abdominal aneurysm presents with minor GI bleeding, visualizing the full length of distal duodenum is essential. Diagnostic modality of choice is intravenous contrastenhanced CT, which has a sensitivity of up to $94 \%$ and specificity of up to $85 \%$ in detecting AEF (5). Other diagnostic modalities have a low diagnostic value and include esophagogastroduodenoscopy, conventional angiography, technetium scan, and enteroclysis. Angiography should be performed during active bleeding and may even accelerate death by displacing the cap thrombus in the fistula tract (5). In our case, initial presentation findings were vague with symptom of abdominal pain and laboratory findings of an inflammatory process. A history of femoral by-pass surgery was a clue to a vascular pathology and hence, contrastenhanced CT was performed.

\subsection{Computed Tomography Findings}

Diagnosis of AEF by CT can be difficult because of subtle findings and potential mimickers (6). Although CT findings of ectopic gas around aorta and contrast extravasation into duodenum are pathognomonic features of AEF, they can be absent. More subtle findings of bowel wall thickening over the aorta, or disruption of the fat covering aorta could be the only clue for the diagnosis (7). In our case, CT images were obtained at different time points during the progression of AEF and after intervention. Initial CT finding was a mural thrombus in the extension of an enhancing soft tissue density that was extending towards the duodenum. The soft tissue density was partially adherent to duodenum and was slightly compressing it. When the patient presented with GI bleeding, CT revealed the pathognomonic features of air bubbles within thrombus along with increased thrombus size and compression of duodenum. Evolution of CT findings indicates that AEF is a progressive process, where adherence and compression leads to fistula formation after an interval. Therefore, in the absence of presenting symptoms like GI bleeding in a patient with abdominal aneurysm, CT alone cannot exclude AEF. Any finding of adherence and compression between an aneurysmatic aortic and enteric segment may lead to fistula formation. A high index of suspicion should be held for a diagnosis of AEF in such cases. Air bubbles, edema, and fluid collection in perigraft region are normal after aortic surgery up to three or four weeks (8). After EVAR treatment of the case, control CT images revealed an increase in air bubbles within thrombus, possibly secondary to surgery. Prompt diagnosis of AEF is essential for a timely intervention to avoid high mortality rates. Achieving a diagnosis can be difficult because of atypical clinical presentation and subtle imaging findings. A high level of suspicion for AEF should be held in any patient with an aneurysmatic aortic segment adherent to duodenum, even without the typical findings of air bubbles.

\section{Acknowledgements}

None declared.

\section{Authors' Contributions}

Furuzan Numan, Fatih Gulsen and Ahmet Bas per- 
Bas A et al.

formed endovascular aneurysm repair. Sedat Giray Kandemirli interpreted the computed tomography images. Ahmet Bas, Osman Simsek, Babak Rafiee and Sedat Giray Kandemirli wrote the manuscript and Furuzan Numan acted as a guide.

\section{Financial Disclosure}

Authors stated that they had no financial interests related to the material in the manuscript.

\section{Funding/Support}

None declared.

\section{References}

1. Katsinelos P, Paroutoglou G, Papaziogas B, Beltsis A, Mimidis K, Pilpilidis I, et al. Secondary aortoduodenal fistula with a fatal outcome: report of six cases. Surg Today. 2005;35(8):677-81.

2. Lemos DW, Raffetto JD, Moore TC, Menzoian JO. Primary aortoduodenal fistula: a case report and review of the literature. J Vasc Surg. 2003;37(3):686-9.

3. Peirce RM, Jenkins RH, Maceneaney P. Paraprosthetic extravasation of enteric contrast: a rare and direct sign of secondary aortoenteric fistula. AJR Am J Roentgenol. 2005;184(3 Suppl):S73-4.

4. Mehmood RK, Mushtaq A, Andrew DR, Miller GA. Clinical presentation of a missed primary aorto-enteric fistula. J Pak Med Assoc. 2007;57(12):616-8.

5. Wood A, Bendjelid SM, Bendjelid K. Primary aortoenteric fistula: should enhanced computed tomography be considered in the diagnostic work-up? Anesth Analg. 2005;101(4):1157-9.

6. Raman SP, Kamaya A, Federle M, Fishman EK. Aortoenteric fistulas: spectrum of CT findings. Abdom Imaging. 2013;38(2):367-75.

7. Lee JT, Saroyan RM, Belzberg G, Pianim NA, Bongard FS. Primary aortoenteric fistula: computed tomographic diagnosis of an atypical presentation. Ann Vasc Surg. 2001;15(2):251-4.

8. Marolt U, Potrc S, Bergauer A, Arslani N, Papes D. Aortoduodena fistula three years after aortobifemoral bypass: case report and literature review. Acta Clin Croat. 2013;52(3):363-8. 